\title{
Examination of a distress screening intervention for rural cancer survivors reveals low uptake of psychosocial referrals
}

\author{
Pam Baker DeGuzman ${ }^{1,2}$ (D) David L. Vogel ${ }^{3} \cdot$ Bethany Horton $^{4} \cdot$ Veronica Bernacchi ${ }^{1} \cdot$ C. Allen Cupp ${ }^{2}$. \\ B. J. Ferrebee Ghamandi ${ }^{5}$. Ivora D. Hinton ${ }^{1}$ • Christi Sheffield ${ }^{2} \cdot$ Mark J. Jameson $^{2,5}$
}

Received: 7 November 2020 / Accepted: 26 April 2021 / Published online: 13 May 2021

(C) The Author(s), under exclusive licence to Springer Science+Business Media, LLC, part of Springer Nature 2021

\begin{abstract}
Purpose To determine the impact of a telemedicine-delivered intervention aimed at identifying unmet needs and cancer-related distress (CRD) following the end of active treatment on supportive care referral patterns.

Methods We used a quasi-experimental design to compare supportive care referral patterns between a group of rural cancer survivors receiving the intervention and a control group $(N=60)$. We evaluated the impact of the intervention on the number and type of referrals offered and whether or not the participant accepted the referral. CRD was measured using a modified version of the National Comprehensive Cancer Network Distress Thermometer and Problem List.

Results Overall, $30 \%$ of participants received a referral for further post-treatment supportive care. Supporting the benefits of the intervention, the odds of being offered a referral were 13 times higher for those who received the intervention than those in the control group. However, even among the intervention group, only $28.6 \%$ of participants who were offered a referral for further psychosocial care accepted.

Conclusions A nursing telemedicine visit was successful in identifying areas of high distress and increasing referrals. However, referral uptake was low, particularly for psychosocial support. Distance to care and stigma associated with seeking psychosocial care may be factors. Further study to improve referral uptake is warranted.

Implications for Cancer Survivors Screening for CRD may be inadequate for cancer survivors unless patients can be successfully referred to further supportive care. Strategies to improve uptake of psychosocial referrals is of high importance for rural survivors, who are at higher risk of CRD.
\end{abstract}

Keywords Cancer survivorship $\cdot$ Rural health $\cdot$ Health disparities $\cdot$ Telemedicine $\cdot$ Referral $\cdot$ Distress screening $\cdot$ Cancer-related distress

\section{Background}

All cancer survivors experience some level of cancer-related distress (CRD; the multifactorial, unpleasant, emotional experience that interferes with survivors' ability to cope with

Pam Baker DeGuzman

deguzman@virginia.edu

1 University of Virginia School of Nursing, PO Box 800782, Charlottesville, VA, USA

2 University of Virginia Cancer Center, Charlottesville, VA, USA

3 Department of Psychology, Iowa State University, Ames, IA, USA

4 Department of Public Health Sciences, University of Virginia School of Medicine, Charlottesville, VA, USA

5 Department of Otolaryngology - Head and Neck Surgery, University of Virginia School of Medicine, Charlottesville, VA, USA cancer, treatment, and symptoms effectively) [1]. Supportive care referrals can be made to services such as physical therapy, social work, or pain management to help with CRD [2]. In the absence of interventions aimed at improving identification of CRD, fewer than $10 \%$ of cancer survivors are referred for psychosocial help [1], although identification of distressed patients and referral rates are far higher when screening tools are routinely used $[3,4]$. Despite interventions designed to screen and refer individuals with high levels of CRD, uptake of these referrals is limited, typically accepted by only onequarter of participants identified as needing further care $[5,6]$.

More rural cancer survivors live with CRD than their urban counterparts [7]. These differences are likely driven by difficulties rural survivors experience accessing post-treatment care. Survivors who have to travel to receive treatment from a distant cancer site may be less willing to travel long distances to receive supportive care [8-10]. The reluctance to travel to non-cancer treatment care is understandable given 
that traveling long distances can be physically painful for a cancer survivor [11]. Disparities created by distance to care are further complicated by social determinants of health: in the USA, rural survivors are more likely to be unemployed due to health reasons. They have lower incomes, lower educational levels, and lower rates of health insurance coverage than their urban counterparts [7]. These disparities can heighten the impact of factors such as the cost of gas and the financial burden incurred by family members to transport family members to appointments [11].

Ideally, rural primary care providers (PCPs) can help evaluate and connect survivors with appropriate care to address CRD; however, PCPs lack the comprehensive training needed to evaluate post-treatment care needs for survivors of an array of complex cancers [12, 13]. A landmark 2005 Institute of Medicine (now National Academy of Medicine) report suggested that all survivors be provided with a comprehensive follow-up plan called a Survivorship Care Plan (SCP) written by the cancer care provider. The SCP is designed to help the PCP and survivor navigate post-treatment health and supportive care needed. Since the 2005 report however, cancer care institutions have struggled with producing and implementing meaningful SCPs, such that the American College of Surgeons Commission on Cancer recently altered requirements for institutions to produce SCPs [14]. Further complicating post-treatment care guidance, rural PCPs may manage health for survivors who may have received cancer care from different treatment centers, so even if they are receiving SCPs, the design and information included in each document may be highly variable, making them difficult to follow [15].

Survivors living a long distance from sites of cancer care may be further disadvantaged because of the difficulty involved in coordinating the optimal time of care delivery with the rural survivor's travel limitations. The end of initial, active treatment can be a common time to provide survivors with education about self-care during the post-treatment survivorship phase [16, 17], particularly for those who have difficulty traveling to return to the original site of care [18]. Research also suggests that a one-time evaluation and SCP delivery at the conclusion of treatment is insufficient [19] and that continuing to provide targeted survivorship education and evaluation into the post-treatment follow-up period may be warranted $[17,20]$.

Rural survivors of head and neck cancer (HNC) particularly vulnerable to having $\mathrm{CRD}$ remain unaddressed. $\mathrm{HNC}$ is the 7th most common cancer worldwide, and the 9th most common in the USA. HNC is considered the most emotionally distressing of all cancers because of the severe consequences on physical integrity and basic functioning, highly visible parts of the body impacted, including those imperative for speech and swallowing [21]. Treatment impacts critical activities such as speech and swallowing, can drastically alter facial integrity, and can lead to long-term pain [22]. A high percentage of long-term post-treatment HNC survivors continue to experience symptoms including dry mouth, taste changes, fatigue, pain, and difficulty in swallowing well into extended survivorship [23, 24]. Nearly three-quarters of HNC survivors have unmet needs during their survivorship trajectory, [25] and the majority of unmet needs are psychological [26]. Unmet needs extend well into the post-treatment phase of HNC survivorship; researchers have found an average of 3.7 unmet needs in survivors $4-5$ years following treatment completion [25]. The impact of unmet needs on quality of life is significant in this population; rurality further heightens the impact. A study of 285 post-treatment HNC survivors found that travel distance to the primary treatment site was found to be independently associated with more unmet needs and poorer quality of life (QoL) during survivorship [23].

We developed and implemented an intervention to identify specific drivers of CRD in HNC survivors, targeted at identifying unmet needs and making referrals for further supportive care in the period following the end of active treatment. The intervention was delivered using a telemedicine video visit (VV) to reduce the need for survivors to travel long distances. The purpose of this study was to determine the impact of a telemedicine-delivered $\mathrm{HNC}$-focused $\mathrm{CRD}$ intervention on supportive care referral patterns.

\section{Methods}

We used a quasi-experimental design to accomplish the study aim. We delivered the intervention to 30 rural HNC survivors, and data from a control group of 30 rural $\mathrm{HNC}$ survivors was extracted from the EMR. Intervention participants were recruited from the Head and Neck Cancer Clinic at the University of Virginia Cancer Center, a National Cancer Institute-designated Cancer Center in the Southeast United States, to participate in a nurse-led, telemedicine-delivered intervention aimed at identifying and addressing CRD posttreatment. The University of Virginia Institutional Review Board approved the study and provided oversight for human subjects' protection.

\section{Intervention}

The intervention is based on Jefford and colleagues' face-toface in-person nurse-led end-of-treatment (EOT), survivorship-focused visit, utilizing distress screening, prioritization of concerns, education, and referral for cancer survivors [27]. We adapted the nurse visit by moving the in-person visit from co-occurring with the EOT visit, to take place in the time period following conclusion of active treatment, and by developing an assessment (see "Assessment", below) to specifically target areas of distress encountered by HNC survivors. The intervention was delivered via a telemedicine VV, in 
order to support a rural cohort. The adapted intervention is called Comprehensive Assistance: Rural, Interventions, Nursing, and Guidance (CARING). Delivery of CARING using the telemedicine modality has been described previously [18].

Figure 1 contains an overview of the intervention, including integration into medically focused follow-up care. In this clinical practice, patients receive SCPs at the EOT visit, which is followed by a medical follow-up visit 3 months later. Participants were scheduled for a telemedicine with the nurse approximately 6 weeks following the EOT visit. The intervention utilizes distress assessment, targeted education, and referral to supportive care resources, when indicated, delivered by an HNC-specialized oncology registered nurse.

Assessment CRD assessment is conducted by the oncology nurse using a modified version of the National Comprehensive Cancer Network (NCCN) Distress Thermometer and Problem List (DT). The DT tool is intended for the clinical setting, to evaluate survivors' current distress around 5 domains: practical problems, family problems, emotional problems, spiritual and religious concerns, and physical problems. Patients rate distress on an 11-point scale from 0 (no distress) to 10 (extreme distress) [28]. The tool is typically used as a single-item rating with patients indicating which areas of distress they are currently experiencing. We amended the tool to include problems typically experienced by HNC survivors: jaw swelling, speech, and hearing [29]. Rather than rate only overall distress, the nurse verbally listed each area of potential distress and asked the participant to rate each area on the 11-point scale (see Supplementary Material). Utilizing this data as a starting point, the nurse led a discussion with the survivor to investigate and prioritize the most distressing issues.

Education Based on issues identified in the DT screening and discussion, the nurse provided targeted survivorship education. Participants who were experiencing high rates of distress (identified either as $\geq 4 / 10$ on an item or through the nursepatient discussion) received education targeted toward their specific area(s) of distress. When the nurse assessment revealed that participants were successfully managing their distress, or if it had already resolved, they focused education on the SCP that was presented at the EOT visit, including cancer surveillance and health promotion, per American Cancer Society recommendations [30].

Referral The last component of the visit is intended to connect survivors experiencing high distress with referrals for further supportive care. Utilizing data gathered during the assessment and education components, the nurse identified remaining areas of distress not resolved through the discussion and education. The nurse offered the referrals to the participant, who either accepted or declined the referral.

\section{Data collection and analysis}

Intervention group For each telemedicine visit conducted, the nurse documented the education provided, types of referrals offered, and whether or not the participated accepted or declined the referral. Cancer site, type, and stage were extracted from the electronic medical record (EMR). Stage was classified as either early (no evidence of metastasis) or late (nodal or distant involvement).

Nursing documentation was reviewed to determine each participant's level of self-reported distress using the amended DT. To classify the amount of high distress (HD) being experienced by each participant, we identified how many areas each participant rated as 4 or higher. We totaled the scores of all areas of HD for each participant and termed this their Distress Score.

Education type was categorized as education only for those identified by the nurse as not in need of further referral or education and referral for those who were offered a referral to further support. We categorized all referrals as either physical support (including speech language pathology, nutrition,
Fig. 1 Overview of the Comprehensive Assistance: Rural Intervention, Nursing, and Guidance (CARING) intervention

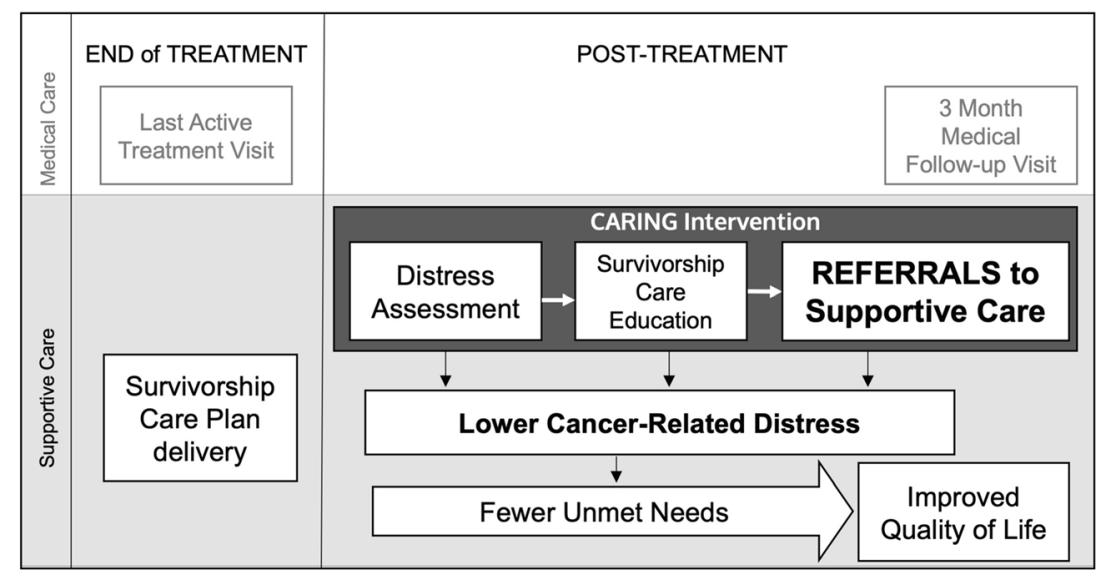


and physical therapy) or psychosocial support (including social work and financial counseling) and determined the number and type of referrals offered at each visit.

Control We sampled 30 sequential records from the EMR of patients who visited the HNC clinic during the midpoint of the intervention, beginning with August 2019. Control group inclusion was identical to CARING participants except that patients were excluded if they had participated in CARING. Control group participants were head and neck cancer survivors who received usual survivorship care at the cancer clinic. They did not receive the telemedicine intervention. Usual care for assessing survivorship needs and distress are determined by each provider. We determined the EOT date; audited the EMR for any referrals offered, accepted, and declined during the 3 months following the EOT visit; and classified them as either physical care or psychosocial care. We recorded cancer site, type, and stage similar to the intervention arm.

Data analysis Logistic regression was used to model references offered. The Firth penalized likelihood approach was used to stabilize the model estimates due to sparse data when certain categorical covariates were considered [31]. In order to build a model of referrals offered, univariate logistic regression models were initially considered. Covariates considered in the univariate models include study arm (intervention vs. usual care), age, race, ethnicity, and stage. For statistical tests comparing variables presented in Tables 1 and 3 by treatment arm, Fisher's exact test was used for categorical variables, and the Wilcoxon-Mann-Whitney test was used for continuous variables. All statistical analyses were performed using SAS (version 9.4, SAS Institute Inc, Cary, NC).

\section{Results}

Forty intervention participants were consented and enrolled in the intervention part of the study, 30 of whom completed the intervention $(75 \%)$. There were no statistical differences in age, race, ethnicity, or home-based broadband between those who received the intervention and those who did not. Table 1 contains demographic characteristics of intervention and control group participants and distress characteristics of intervention participants. The majority of both intervention and usual care participants were Non-Hispanic White male, with an average age of 61.3 and 63.3 years of age for intervention and usual care participants, respectively. These characteristics are similar to the population of rural patients with $\mathrm{HNC}$ seen at the practice setting. The most common cancer sites were oral cavity, thyroid, pharynx, and larynx, although proportions differed between the groups. More than half of participants did not have nodal involvement or metastases, with similar proportions between groups.
All 30 intervention participants self-reported at least one area of distress, and 27 of the 30 had at least one area of HD (90.0\%). The mean Distress Score for those experiencing HD was 37.0 .

Table 2 presents odds ratios and $95 \%$ confidence intervals for participants being offered a referral for post-treatment supportive care. Only participation in the intervention arm was a significant predictor of being offered a referral, with the odds of being offered a referral 13 times higher for those who received the intervention than those in the control group.

Table 3 presents patterns of referrals offered and accepted for those in the intervention and control groups. Eighteen $(30 \%)$ of the participants received a referral for further supportive care, with over half of intervention participants being offered a referral vs. just 2 of those in the control group ( $p=$ 0.0001 ). Differences in referral patterns between the intervention and control groups were similarly reflected; just $3.3 \%$ of control group participants were offered a referral for further psychosocial care vs. $46.7 \%$ of those in the intervention group ( $p=0.0002$ ), and $3.3 \%$ of control group participants were offered a referral for further physical care vs. $10 \%$ of intervention group participants $(p=0.6120)$. Overall, $15 \%$ of all participants agreed to accept a referral for further care. Accepted referrals trended higher among intervention group participants (23.3\% vs. $6.7 \%, p=0.1455)$.

Of all 18 participants offered any type of referral, $9(50 \%)$ accepted. In the control group, both participants offered a referral for further care accepted it (one physical referral and one psychosocial referral). Among intervention participants, $7 / 16(43.8 \%)$ of participants accepted a referral; however, while all three physical referrals were accepted, only $4 / 14$ (28.6\%) participants who were offered a referral for further psychosocial care accepted.

\section{Discussion}

Our innovative approach to delivering post-treatment cancer care was successful in identifying rural $\mathrm{HNC}$ survivors experiencing HD. Of the rural HNC survivors who participated in our telemedicine-delivered distress screening intervention, most identified at least one area of $\mathrm{HD}$, a finding consistent with prior studies $[5,6,10]$. Participation in the intervention also led to a significantly higher rate of being offered a referral. In fact, over half of all intervention participants were offered a referral, compared with fewer than $10 \%$ of control group participants. Referrals, specifically those made for psychosocial care, followed a similar pattern: nearly half of intervention participants were offered a post-treatment referral compared with just $3.3 \%$ of control group participants. Our control group findings are consistent with prior research that has found fewer than $10 \%$ of survivors with significant distress are identified and referred for further psychosocial help 
Table 1 Participant characteristics $(n=60)$

\begin{tabular}{|c|c|c|c|c|}
\hline Characteristic & All & Intervention $(n=30)$ & Usual care $(n=30)$ & $p$-value \\
\hline \multicolumn{5}{|l|}{ Gender } \\
\hline Male & $38(63.3)$ & $17(56.7)$ & $21(70.0)$ & 0.42 \\
\hline Female & $22(36.7)$ & $13(43.3)$ & $9(30.0)$ & \\
\hline Age (mean; sd; range) & $62.3(12.0 ; 33-88)$ & $61.3(13.9 ; 33-88)$ & $63.3(9.9 ; 48-85$ & 0.83 \\
\hline Race & & & & 0.93 \\
\hline White & $48(80.0)$ & $25(83.3)$ & $23(76.7)$ & \\
\hline Black & $6(10.0)$ & $2(6.7)$ & $4(13.3)$ & \\
\hline Asian & $2(3.3)$ & $1(3.3)$ & $1(3.3)$ & \\
\hline Other & $4(6.7)$ & $2(6.7)$ & $2(6.7)$ & \\
\hline Ethnicity & & & & 1.0 \\
\hline Non-Hispanic & $57(95.0)$ & $28(93.3)$ & $29(96.7)$ & \\
\hline Hispanic & $3(5.0)$ & $2(6.7)$ & $1(3.3)$ & \\
\hline Cancer site & & & & 0.03 \\
\hline Oral cavity & $29(48.3)$ & $10(33.3)$ & $19(63.3)$ & \\
\hline Thyroid & $12(20.0)$ & $9(30.0)$ & $3(10.0)$ & \\
\hline Pharynx & $8(13.3)$ & $6(20.0)$ & $2(6.7)$ & \\
\hline Larynx & $4(6.7)$ & $1(3.3)$ & $3(10.0)$ & \\
\hline Other & $6(10.0)$ & $3(10.0)$ & $3(10.0)$ & \\
\hline More than one site & $1(1.7)$ & $1(3.3)$ & $0(0.0)$ & \\
\hline Cancer type & & & & 0.07 \\
\hline Squamous cell carcinoma & $42(70.0)$ & $18(60.0)$ & $24(80.0)$ & \\
\hline Papillary thyroid carcinoma & $12(20.0)$ & $9(30.0)$ & $3(10.0)$ & \\
\hline Other & $6(10.0)$ & $3(10.0)$ & $3(10.0)$ & \\
\hline Cancer stage & & & & 1.0 \\
\hline Early & $39(65.0)$ & $20(66.7)$ & $19(63.3)$ & \\
\hline Late & $21(35.0)$ & $10(33.3)$ & $11(36.7)$ & \\
\hline Distress Score $(N=27)$ & $*$ & & $*$ & $*$ \\
\hline (mean; sd; range) & & $37.0 ; 30.7 ; 7-165$ & & \\
\hline
\end{tabular}

*Distress data only collected for intervention group
Table 2 Odds ratios and 95\% confidence intervals for participants being offered a referral for post-treatment supportive care

\begin{tabular}{lll}
\hline Characteristic & Odds ratio & $95 \%$ CI \\
\hline Arm & & \\
Control & Ref. & \\
Intervention & 13.0 & $3.4-72.2$ \\
Gender & & \\
Male & Ref. & \\
Female & 2.2 & $0.7-6.7$ \\
Race & & \\
White & Ref. & \\
Black & 1.2 & $0.2-6.1$ \\
Asian & 0.4 & $0.0-5.7$ \\
Other & 0.9 & $0.1-6.2$ \\
Ethnicity & & \\
Non-Hispanic & Ref. & $0.5-48.4$ \\
Hispanic & 4.2 & \\
Cancer stage & & \\
Late & Ref. & \\
Early & 2.2 & $0.7-8.2$ \\
\hline
\end{tabular}

[1], furthering evidence of the need to screen and make referrals for cancer-related distress in the post-treatment period. Offers for further psychosocial care were high across the entire sample, accounting for 15 referrals made to the 19 patients offered a referral; the relatively lower number of physical care referrals may reflect a likelihood of physical issues having already been addressed in survivors' routine follow up visits with the physician [32].

\section{Referral uptake}

Among all referrals, half were accepted. All referrals offered to control group participants were accepted; however, only two had been offered. In this HNC practice, usual care typically involves survivors initiating a request for post-treatment referrals, as such these two referrals were survivor-initiated, and thus unsurprising that both were accepted. Additionally, $100 \%$ of referrals offered for physical care were accepted, regardless of group. 
Table 3 Patterns of supportive care referrals offered and accepted as a proportion of total participants $(n=60)$

\begin{tabular}{|c|c|c|c|c|c|c|c|c|}
\hline & \multicolumn{4}{|c|}{ Referrals offered } & \multicolumn{4}{|c|}{ Referrals accepted } \\
\hline & $\begin{array}{l}\text { All } \\
(n=60)\end{array}$ & $\begin{array}{l}\text { Intervention } \\
(n=30)\end{array}$ & $\begin{array}{l}\text { Control } \\
(n=30)\end{array}$ & $p$-value & $\begin{array}{l}\text { All } \\
(n=60)\end{array}$ & $\begin{array}{l}\text { Intervention } \\
(n=30)\end{array}$ & $\begin{array}{l}\text { Control } \\
(n=30)\end{array}$ & $p$-value \\
\hline All referrals & $18(30.0)$ & $16(53.3)$ & $2(6.7)$ & 0.0001 & $9(15.0)$ & 7 (23.3) & $2(6.7)$ & 0.1455 \\
\hline Psychosocial referrals & $15(25.0)$ & $14(46.7)$ & $1(3.3)$ & 0.0002 & $5(8.3)$ & $4(13.3)$ & $1(3.3)$ & 0.3533 \\
\hline Physical referrals & $4(6.7)$ & $3(10.0)$ & $1(3.3)$ & 0.6120 & $4(6.7)$ & $3(10.0)$ & $1(3.3)$ & 0.6120 \\
\hline
\end{tabular}

Note: One intervention patient was offered both a physical and psychosocial referral, so the total of psychosocial and physical referrals $>$ all referral

The rate of accepted psychosocial referrals among intervention participants was low, although not surprising at $28.6 \%$. It is possible that participants with HD felt that they had gained sufficient support via the targeted distress discussion and education with the nurse. However, previous research indicates that interventions designed to screen individuals with cancer for high levels of CRD have generated similarly low uptake of psychosocial referrals $[5,6]$, and rural cancer survivors reluctance to seek supportive care has been well established $[8,9,33,34]$. Still, this low psychosocial referral uptake among survivors experiencing high levels of CRD is concerning. More work is needed to find ways to connect rural survivors with appropriate post-treatment psychosocial intervention; the risk of not connecting rural survivors with support is too high. Individuals with cancer have a fourfold increase in risk of suicide compared to the general U.S. population, with White males, and those with HNC, and cancers of the lung, testes, bladder, and Hodgkin's lymphoma all increasing one's risk [35]. The risk is further compounded for U.S. rural residents, who have significantly higher suicide rate than their urban counterparts [36].

Connecting survivors with appropriate post-treatment care psychosocial care must involve understanding reasons for referral refusal. Rural survivors experiencing high distress may decline post-treatment supportive for a variety of reasons, the most widely researched being distance to care [8-10]. Given our rural demographic, we also believe an important component of refusal was also related to stigma, which has been found to more prevalent among those from U.S. rural communities $[37,38]$. The stigma associated with seeking psychosocial help is the view that a person who seeks treatment is less socially acceptable [39]. Prior research has consistently shown that stigma is associated with more negative attitudes toward and lower intentions to seek help for psychosocial concerns [40-42]. As such, future research could directly examine the effects of stigma on survivors' acceptance of psychosocial care as well as potential ways to reduce it.

Telemedicine offers a mechanism to connect rural survivors with supportive care providers that may address both distance to care and stigma concerns. The current intervention was conducted largely prior to the onset of the coronavirus pandemic of 2019 (COVID19). As such, we offered this screening via telemedicine, but subsequent telemedicine-delivered referring provider visits were not offered during this time. Changes to the availability of VV in healthcare are rapidly developing, and thus, future intervention efforts could offer psychosocial telehealth options. Prior to COVID-19, VVs were less integrated into the care environment, but COVID-19 has catapulted their use into the forefront of American healthcare [43, 44]. However, it is important to note that the limited reach of fixed broadband into the rural USA restricts the ability of some cancer support providers to equitably provide digitally delivered care to rural residents using a modality that limits COVID-19 exposure [18].

\section{Limitations}

The interpretability of our study is limited by several factors. The small number and homogeneity of participants in our study limit the generalizability of our sample, which was conducted in one clinical service in one cancer center in the Southeastern United States. We limited our study of referral acceptance to information found in the EMR during the 3 months following EOT. For those participants who accepted a referral, we did not follow them to determine if they ultimately accepted psychosocial help. Our control group was selected sequentially rather than randomly, which may have resulted in a biased sample, as represented by differences in cancer type and site, which may have contributed to differences in referral uptake between the groups. We also utilized the DT assessment in a unique way, to initiate a discussion of specific areas of CRD with our patients. This method of DT use has not been evaluated for its validity, and thus, the rate of identification of HD in our study cannot be compared to other research using the DT. Further research is needed to determine if this is a valid method of measuring CRD in a clinical cancer population. Finally, the drop-out rate of our study was high, at $25 \%$. Recruitment and retention of 
rural populations into clinical research have been historically acknowledged as highly challenging by investigators, leading to the underrepresentation of this rural populations in medical research [45]. Accordingly, studies with rural populations are often plagued by similarly high drop-out rates [46, 47].

\section{Conclusions}

Screening for CRD may be inadequate for cancer survivors unless patients can be referred to further supportive care. Uptake of psychosocial referral is of high importance for rural survivors, who are at higher risk of having their needs left unmet following active treatment. Interventions to improve referral uptake in cancer survivors need to consider offering $\mathrm{VV}$ for all types of care, but challenges in broadband access must be addressed. Further development and testing of interventions to improve referral uptake are warranted.

Supplementary Information The online version contains supplementary material available at https://doi.org/10.1007/s11764-021-01052-4.

Acknowledgements The authors would like to acknowledge the University of Virginia Karen S. Rheuban Center for Telehealth for its support of this project.

Author contribution PD conceived and designed the study, acquired and interpreted the data, and drafted and revised the work. DV interpreted the data and drafted and revised the work. BH analyzed and interpreted the data and drafted and revised the work. VB, CAC, BJFG, IDH, and CS acquired and interpreted the data and revised the work. MJJ conceived and designed the study, acquired and interpreted the data, and revised the work. All authors approved the version to be published and agree to be accountable for all aspects of the work in ensuring that questions related to the accuracy or integrity of any part of the work are appropriately investigated and resolved.

Funding This study was supported by a grant from the University of Virginia Cancer Center Cancer.

Data availability We do not have permission to share this data outside of the research team

\section{Declarations}

Ethics approval Approved by the University of Virginia Health Sciences Research IRB

Consent to participate Verbal informed consent was obtained from all intervention participants prior to the intervention and any data collection.

Consent for publication Verbal informed consent was obtained from all intervention participants prior to the intervention and any data collection.

Conflict of interest The authors declare no competing interests.

\section{References}

1. Holland JC, Andersen B, Breitbart WS, Buchmann LO, Compas B, Deshields TL, et al. Distress management. J Natl Compr Cancer Netw. 2013;11(2):190-209.

2. Hodgkinson K, Butow P, Hunt GE, Pendlebury S, Hobbs KM, Lo SK, et al. The development and evaluation of a measure to assess cancer survivors' unmet supportive care needs: the CaSUN (Cancer Survivors' Unmet Needs measure). Psychooncology. 2007;16(9): 796-804. https://doi.org/10.1002/pon.1137.

3. Lee J, Jung D, Kim W, Lee H, Noh D, Hahm B. Correlates of oncologist-issued referrals for psycho-oncology services: what we learned from the electronic voluntary screening and referral system for depression (eVSRS-D). 2016;178(June 2015):170-178.

4. Skaczkowski G, Sanderson P, Shand M, Byrne A, Wilson C. Factors associated with referral offer and acceptance following supportive care problem identification in a comprehensive cancer service. Eur J Cancer Care (Engl). 2018;27(5):1-11. https://doi.org/ 10.1111/ecc. 12869

5. Funk R, Cisneros C, Williams RC, Kendall J, Hamann HA. What happens after distress screening? Patterns of supportive care service utilization among oncology patients identified through a systematic screening protocol. Support Care Cancer. 2016;24(7):2861-8. https://doi.org/10.1007/s00520-016-3099-0.

6. Bauwens S, Baillon C, Distelmans W, Theuns P. Systematic screening for distress in oncology practice using the Distress Barometer: the impact on referrals to psychosocial care. Psychooncology. 2014;23(7):804-11. https://doi.org/10.1002/pon. 3484.

7. Weaver KE, Geiger AM, Lu L, Case LD. Rural-urban disparities in health status among US cancer survivors. Cancer. 2013;119(5): 1050-7. https://doi.org/10.1002/cncr.27840.

8. Lavergne MR, Johnston GM, Gao J, Dummer TJB, Rheaume DE. Variation in the use of palliative radiotherapy at end of life: examining demographic, clinical, health service, and geographic factors in a population-based study. Palliat Med. 2011;25(2):101-10. https://doi.org/10.1177/0269216310384900.

9. Pesut B, Robinson CA, Bottorff JL, Fyles G, Broughton S. On the road again: patient perspectives on commuting for palliative care. Palliat Support Care. 2010;8(02):187-95. https://doi.org/10.1017/ S1478951509990940.

10. Opie CA, Koschel A, Ervin KE, Jeffreson L, Haines HM. Supportive care screening in rural ambulatory cancer care. Aust J Cancer Nurs. 2017;18(2):3-9.

11. Paul CL, Hall AE, Carey ML, Cameron EC, Clinton-McHarg T. Access to care and impacts of cancer on daily life: do they differ for metropolitan versus regional hematological cancer survivors? J Rural Health. 2013;29 Suppl 1(s1):s43-50. https://doi.org/10. 1111/jrh.12020.

12. McCabe MS, Jacobs LA. Clinical update: survivorship caremodels and programs. Semin Oncol Nurs. 2012;28(3):e1-8. https://doi.org/10.1016/j.soncn.2012.05.001.

13. Hewitt M, Greenfield S, Stovall E, Medicine I of. From cancer patient to cancer survivor: lost in transition. (Hewitt $\mathrm{M}$, Greenfield S, Stovall E, eds.). Washington D.C.: National Academies Press; 2005.

14. American College of Surgeons. Cancer program standards: ensuring patient-centered care. Comm Cancer. 2016;2:1-84. https:// www.facs.org/quality-programs/cancer/coc/standards.

15. Isaacson MJ, Hulme PA, Cowan J, Kerkvliet J. Cancer Survivorship Care Plans: processes, effective strategies, and challenges in a Northern Plains rural state. Public Health Nurs. 2018;35(4):291-8. https://doi.org/10.1111/phn.12393. 
16. Boekhout AH, Maunsell E, Pond GR, et al. A Survivorship Care Plan for breast cancer survivors: extended results of a randomized clinical trial. J Cancer Surviv. 2015;9(4):683-91.

17. DeGuzman PB, Colliton KC, Nail CJ, Keim-Malpass J. Survivorship Care Plans: rural, low-income breast cancer survivor perspectives. Clin J Oncol Nurs. 2017;21(6):6928. http://www.scopus.com/inward/record.url?eid=2-s2.085034982274\&partnerID=MN8TOARS.

18. DeGuzman PB, Bernacchi V, Cupp CA, et al. Beyond broadband: digital inclusion as a driver of inequities in access to rural cancer care. J Cancer Surviv. 2020;14(5):643-52. https://doi.org/10.1007/ s11764-020-00874-y.

19. de Rooij BH, Thomas TH, Post KE, Flanagan J, Ezendam NP, Peppercorn J, \& Dizon DS. Survivorship care planning in gynecologic oncology - perspectives from patients, caregivers, and health care providers. J Cancer Surviv. 2018;12(6):762-774. https://doi. org/10.1007/s11764-018-0713-9

20. Boyajian RN, Grose A, Grenon N, Roper K, Sommer K, Walsh M, et al. Desired elements and timing of cancer survivorship care: one approach may not fit all. J Oncol Pract. 2014;10(5):e293-8. https:// doi.org/10.1200/JOP.2013.001192.

21. Osazuwa-Peters N, Simpson MC, Zhao L, Boakye EA, Olomukoro SI, Deshields T, et al. Suicide risk among cancer survivors: head and neck versus other cancers. Cancer. 2018;124(20):4072-9. https://doi.org/10.1002/cncr.31675.

22. Murphy BA, Ridner S, Wells N, Dietrich M. Quality of life research in head and neck cancer: a review of the current state of the science. Crit Rev Oncol Hematol. 2007;62(3):251-67. https://doi.org/10. 1016/j.critrevonc.2006.07.005.

23. So WKW, Choi KC, Chen JMT, Chan CWH, Chair SY, Fung OWM, et al. Quality of life in head and neck cancer survivors at 1 year after treatment: the mediating role of unmet supportive care needs. Support Care Cancer. 2014;22(11):2917-26. https://doi.org/ 10.1007/s00520-014-2278-0.

24. Cramer JD, Johnson JT, Nilsen ML. Pain in head and neck cancer survivors: prevalence, predictors, and quality-of-life impact. Otolaryngol - Head Neck Surg (U S). 2018;159(5). https://doi. org/10.1177/0194599818783964.

25. Wells M, Cunningham M, Lang H, Swartzman S, Philp J, Taylor L, et al. Distress, concerns and unmet needs in survivors of head and neck cancer: a cross-sectional survey. Eur J Cancer Care (Engl). 2015;24(5):748-60. https://doi.org/10.1111/ecc.12370.

26. Henry M, Habib LA, Morrison M, Yang JW, Li XJ, Lin S, et al. Head and neck cancer patients want us to support them psychologically in the posttreatment period: survey results. Palliat Support Care. $2013 ; 12(6): 481-93$. https://doi.org/10.1017/ S1478951513000771

27. Jefford M, Aranda S, Gough K, et al. Evaluating a nurse-led survivorship care package (SurvivorCare) for bowel cancer survivors: study protocol for a randomized controlled trial. Trials. 2013;14: 260. https://doi.org/10.1186/1745-6215-14-260.

28. Snowden A, White CA, Christie Z, Murray E, McGowan C, Scott $\mathrm{R}$. The clinical utility of the distress thermometer: a review. Br J Nurs. 2011;20(4):220-7. https://doi.org/10.12968/bjon.2011.20.4. 220.

29. Nguyen NTA, Ringash J. Head and neck cancer survivorship care: a review of the current guidelines and remaining unmet needs. Curr Treat Options Oncol. 2018;19(8). https://doi.org/10.1007/s11864018-0554-9.

30. Cohen E, LaMonte S, Erb N, et al. American Cancer Society Head and Neck Cancer Survivorship Care Guideline. CA Cancer J Clin. 2016;66(3):204-39. https://doi.org/10.3322/caac.21343.
31. Firth D. Bias reduction of maximum likelihood estimates. Biometrika. 1993;80:27-38.

32. Franco K, Shuk E, Philip E, Blanch-Hartigan D, Parker PA, Matasar M, et al. Communication between oncologists and lymphoma survivors during follow-up consultations: a qualitative analysis. J Psychosoc Oncol. 2017;35(5):513-30. https://doi.org/10. 1080/07347332.2017.1313352.

33. Nathan PC, Agha M, Pole JD, Hodgson D, Guttmann A, Sutradhar $\mathrm{R}$, et al. Predictors of attendance at specialized survivor clinics in a population-based cohort of adult survivors of childhood cancer. $\mathrm{J}$ Cancer Surviv. 2016;10(4):611-8.

34. Ou JY, Smits-Seemann RR, Wu YP, Wright J, Kirchhoff AC. An investigation of survivorship clinic attendance among childhood cancer survivors living in a five-state rural region. J Cancer Surviv. 2018;12(2):196-205.

35. Zaorsky NG, Zhang Y, Tuanquin L, Bluethmann SM, Park HS, Chinchilli VM. Suicide among cancer patients. Nat Commun. 2019;10(1):1-7. https://doi.org/10.1038/s41467-018-08170-1.

36. Rossen LM, Hedegaard H, Khan D, Warner M. County-level trends in suicide rates in the U.S., 2005-2015. Am J Prev Med. 2018;55(1):72-9. https://doi.org/10.1016/j.amepre.2018.03.020.

37. Jones AR, Cook TM, Wang J. Rural-urban differences in stigma against depression and agreement with health professionals about treatment. J Affect Disord. 2011;134(1-3):145-50.

38. Stewart H, Jameson JP, Curtin L. The relationship between stigma and self-reported willingness to use mental health services among rural and urban older adults. Psychol Serv. 2015;12(2):141-8.

39. Vogel DL, Wade NG, Haake S. Measuring the self-stigma associated with seeking psychological help. J Couns Psychol. 2006;53(3): 325-37. https://doi.org/10.1037/0022-0167.53.3.325.

40. Brenner RE, Cornish MA, Heath PJ, Lannin DG, Losby MM. Seeking help despite the stigma: experiential avoidance as a moderated mediator. J Couns Psychol. 2019.

41. Lannin DG, Vogel DL, Brenner RE, Tucker JR. Predicting selfesteem and intentions to seek counseling: the internalized stigma model. Couns Psychol. 2015;43(1):64-93.

42. Pattyn E, Verhaeghe M, Sercu C, Bracke P. Public stigma and selfstigma: differential association with attitudes toward formal and informal help seeking. Psychiatr Serv. 2014;65(2):232-8.

43. Mann DM, Chen M, Chunara R, Testa P, Nov O. COVID-19 transforms health care through telemedicine: evidence from the field. Digit Econ Glob Margins. 2020. https://doi.org/10.7551/mitpress/ 10890.003.0026.

44. Tanaka MJ, Oh LS, Martin SD, Berkson EM. Telemedicine in the era of COVID-19: the virtual orthopaedic examination. J Bone Joint Surg Am. 2020;102:e57.

45. Tanner A, Kim S-H, Friedman DB, Foster C, Bergeron CD. Barriers to medical research participation as perceived by clinical trial investigators: communicating with rural and African American communities. J Health Commun. 2015;20(1):88-96.

46. McCrone SH. Recruitment and Retention of rural women into research protocols: lessons learned. In: 16 Th International Nursing Research Congress. 2005.

47. Weinert C, Cudney S, Hill W. Retention in a computer-based outreach intervention for chronically ill rural women. Appl Nurs Res. 2008;21(1):23-9.

Publisher's note Springer Nature remains neutral with regard to jurisdictional claims in published maps and institutional affiliations. 